\title{
Analyzing Customer Satisfaction Over Telecom Companies in Sudan Using Apriori Algorithm
}

\author{
Sallam Osman Fageeri ${ }^{1}$, S. Arockiasamy ${ }^{2}$, Aiman Moyaid Said ${ }^{3 *}$ \\ 1,2,3 Department of Information Systems, College of Economics, Management and Information \\ Systems, \\ University of Nizwa, Sultanate of Oman
}

\begin{abstract}
With the rapid growth in the field of information and communication technology, the demand for telecommunications and internet services increased over the years. In Sudan, three reputed telecom companies provide these types of services. Although a great competition between these companies in order to attract more new customers and also to keep the existing customers satisfied, many new and existing customers still has some apprehensions over the difficulty on how to choose the best among the service providers in terms of full range of services with affordable cost. This study aims to measure the customer satisfaction from the telecom companies in Sudan. The survey questionnaire designed and distributed electronically to as many customers to find frequent patterns by applying association rule mining techniques. The results of analysis show that Zain Sudan performs better compared to MTN and Sudani telecom companies in terms of various customer-oriented services.
\end{abstract}

\section{Introduction}

The telecommunication in Sudan started in the year 1897 by the colonization as for phone communication. In Sudan, there are three well-known companies such as ZAIN, MTN, SUDANI, and Zain Sudan works in the field of GSM under the license granted by the national telecommunications authority. It is the first company used 4G LTE network. Today, Zain is proud to own the biggest market share of $42 \%$ with more than 12 million active customers. The coverage reached more than $90 \%$ of the Sudan population. The MTN Sudan started in 2002 with official license, then it secured a permission to use the GSM, and its coverage was $45.3 \%$ of the population at the end of 2007, i.e., 1.7 million. Since 2006, Sudani is the mobile arm of SudaTel Group (STG) of companies in Sudan, and its coverage is 7.2 million.

Sudan has a large population using telecommunications and it contains different companies that provide similar services. Although there is a competition between these companies in the services provided to the customers, the peoples are still complaining about many factors, which affect their decision on how to choose telecom service providers. These companies provide a full service without having multiple sim cards, for which the customers usually complain about problems that are related to quality of the services provided [1]. Customers are confused over the selection of best telecommunication companies by taking in to consideration the cost, assurance and responsiveness of services such as calling, SMS, fast

*Corresponding author: aiman.moyaid@unizwa.edu.om 
internet, and strong signature. Each one of these companies Zain, MTN, and Sudani try to improve their services to attract new customers and to keep their existing one satisfied with their services.

To explore the proposed idea of this research, it is important to use the promising Information Technology trends such as Data Mining. Data mining, also known as Knowledge Discovery in Database (KDD), is one of the current technologies of the information age [1][2]. Data mining can be defined as a process of discovering patterns and trends in large-scale databases, in which the information inferred can be used in decision making [3]. The process can be applied in businesses, scientific and government organizations as well as others, where massive data have been collected and ready for analysis [4] [2].

The rest of this paper is organized as Section II contains the literature reviews related to the existing research works. Section III discusses about the Methodology used. Section IV presents and discusses the Implementation and the obtained results. Finally, this paper provides a conclusion in Section V.

\section{Literature Review}

In this section, some literature related to the proposed research work has been reviewed and discussed.

Authors in [4] analysed and measured the impact of quality of service on customer satisfaction based on functional quality and technical quality perspectives. They have collected feedback from 356 respondents by an online and on-field survey. They had found that network quality, empathy, reliability and assurance have positive impact on customer satisfaction.

Authors in [5] discusses the differences in quality of service provided by companies to customers. They had used SPSS 24 (ANOVA and T-Test) as analytical tools and they found customer loyalty does not play a major role to have large customers. However, it also found that women subscribers have more responsiveness to the value-based offerings and older men subscribers were more satisfied. In addition to that, it is recommended that the managers to focus on customer loyalty.

Data that was collected through the survey from 415 users of telecommunication services (landline telephone, television and internet services), found that the responsiveness and the services of the call centres have the most influence in the customer satisfaction with telecommunications services, in addition to that, offered plans and promotions effecting on a suitable cost-benefit services [6]. Author in [7] collected feedback from 100 respondents through questionnaire, and after the analysis on telecommunication services, they found $75 \%$ of mobile users are much satisfied while $25 \%$ has not expressed any form of dissatisfaction. However, customer satisfaction with service delivery still needs an improvement, such as providing good coverage in the rural areas and the time spent by the customers in the counters to be reduced.

\section{Methodology}

This study is describing and aiming to test the factors that lead to the customer satisfaction for telecommunication company Users in Sudan [8]. A structured questionnaire consisting of many dimensions of assurance, responsiveness, and cost were used for data collection [9]. The questionnaire consists of eight sections including 24 questions about customer satisfaction, 104 questions related to quality of services. The customers response are based on the company which provides various services (calls, internet [4]). An online survey has been used to collect feedback from 440 responders in 7 days and this survey has been distributed in several parts of Sudanese telecommunication company services MTN, Zain and Sudani[4]. The customer satisfaction in Telecommunication Companies in Sudan has 
been analysed using the application of data mining techniques from the collected data. The figure 1 shows the overall research framework. Apriori algorithm has been proposed to use for analysing the data and the set of procedure to be followed in data mining is presented in the subsequent sections.

\subsection{Data Pre-Processing}

The preprocessing step involves data cleaning and noise removal. Additionally, it includes collecting required information from selected dataset, providing appropriate strategies for coping with missing data and eliminating redundant data. The pre-processing and transformations of data are the real dataset for analyzing the user satisfaction.

\subsection{Analysis and Implementation}

After comprehensive survey conducted, the data mining techniques such as frequent pattern and association rule mining has been used to extract association rules. The present work intends to use data mining by considering well-known Apriori algorithms. In order to provide a solution for different reasons and factors, association rule mining algorithm is proposed to be used. This is a classic algorithm for mining association rule and used to get frequent item sets from the database, frequency measurement with support and association rule with confidence. Apriori algorithm depends on finding frequent item sets, and if the item sets cannot pass the minimum support test then it will fail.[11] .

The problem of mining association rules can be decomposed into two sub problems: Finding all sets of items (item set) having support which is greater than the user-specified minimum support value. Item sets with minimum support are called frequent item sets. All other items are said to be infrequent items. The support Sup of an item (item set) is calculated using equation (1).

$$
\operatorname{Sup}(X \Rightarrow Y, D)=\frac{\operatorname{Sup}(X U Y, D)}{|D|}
$$

To extract the association rules, the rules that have minimum confidence greater than the user-specified minimum confidence and from the frequent item set. The confidence between two items (item sets) is calculated using equation (2).

$$
\operatorname{Conf}(X \Rightarrow Y, D)=\frac{\operatorname{Sup}(X U Y, D)}{\operatorname{Sup}(X, D)}
$$

Frequent patterns and association rule mining algorithms can be categorized in two main categories [1]:

$>$ Apriori and Apriori-like algorithms.

$>$ Frequent Pattern (FP)-Growth and FP-Growth-like algorithms.

Although Apriori and Apriori-like algorithms suffer from two potentially very high computational costs [2]: (1) The cost of candidate item sets generation and testing, and (2) the cost of repeatedly scanning the database transactions. But the objective of this study is how to analyse the customer satisfaction without looking for the execution time and memory usage and the two factors are out of our scope. 


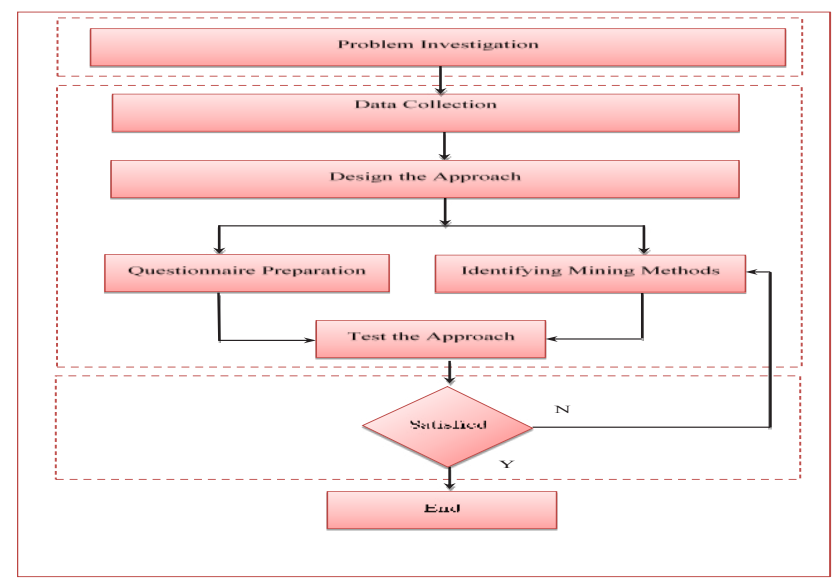

Figure 1: The overall research framework

Based on Apriori algorithm applied below as a sample of frequent item sets. - showing best rules and result found:

Second SIM card $=$ MTN $95 \rightarrow$ first SIM card $=$ ZAIN 67 conf $:(0.71)$

Second SIM card $=$ SUDANI $86 \rightarrow$ first SIM card=ZAIN 54 conf $:(0.63)$

Second SIM card $=$ NULL $174 \rightarrow$ first SIM card $=$ ZAIN 91 conf $:(0.52)$

address $=$ Bahri $137 \rightarrow$ first SIM card $=$ ZAIN 77 conf:(0.56)

address $=$ Khartoum $155 \rightarrow$ first SIM card $=$ ZAIN 80 conf $(0.52)$

address=Omdurman $135 \rightarrow$ first SIM card $=$ ZAIN 66 conf $(0.49)$

period of using=between 6 to 10 years $122 \rightarrow$ first SIM card $=$ ZAIN $72 \operatorname{conf}(0.59)$

period of using=between 1 to 5 years $194 \rightarrow$ first SIM card $=$ ZAIN 92 conf $(0.47)$

first SIM card=MTN $131 \rightarrow$ selection reason $=$ offers and suitable prices 94 conf $(0.72)$

first $\mathrm{SIM}$ card $=$ ZAIN $223 \rightarrow$ selection reason $=$ offers and suitable prices 102 conf $(0.49)$

\section{Implementation, Results and Discussion}

Based on the proposed problem and based on the data collected, it has been analysed using association rule mining techniques and the following results were obtained.

Figure 2 represents the first SIM card that user can use with the additional SIM card. The customer who uses Sudani SIM card and uses ZAIN SIM card as additional SIM is 60\%. The customer who uses MTN SIM card uses as additional card by $30 \%$. The customer, who uses ZAIN SIM card as first sim card, uses MTN SIM card as additional SIM by $50 \%$ and use 


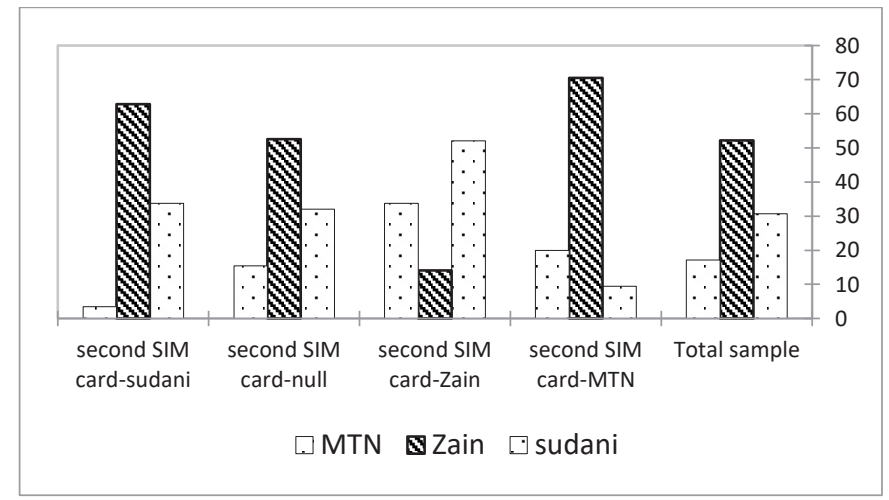

Figure 2. Using of two different SIM cards

Sudani SIM card by $30 \%$. The customer who uses MTN SIM card as first SIM card uses ZAIN SIM card as additional SIM by $70 \%$. After analyzing the data from the total sample, it is concluded that the customers use ZAIN by $52 \%$ MTN by $31 \%$, and Sudani by $17 \%$.

Figure 3 represents the use of the First SIM card with customer's address, and the customers who uses ZAIN SIM card as a first SIM card. Total sample shows the distribution percentage of all customers.

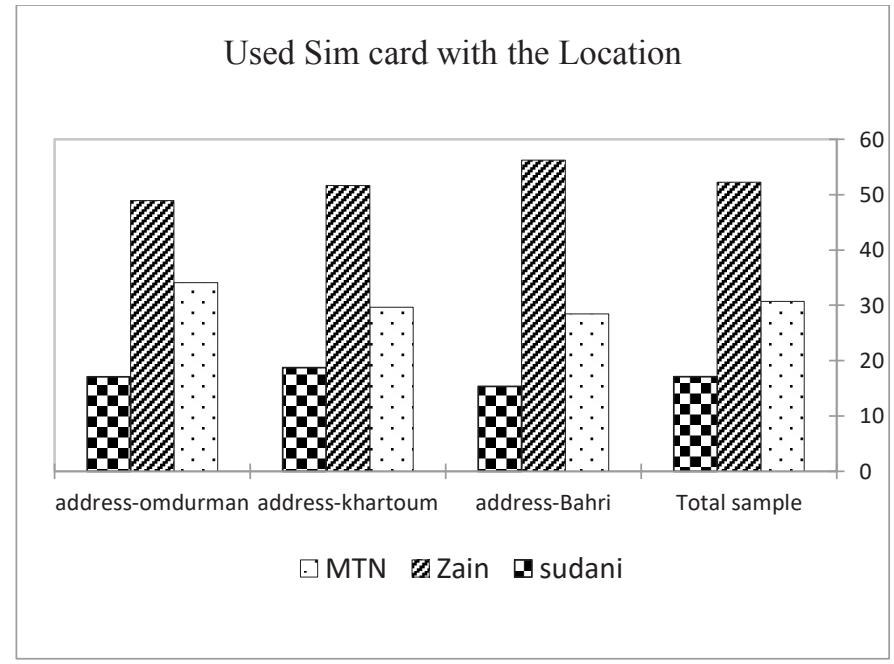

Figure 3: Type of SIM card used in Khartoum state

Figure (4): reveal that the ZAIN SIM card represents long period of use as first SIM card, MTN SIM card as second and Sudani as the last card. This indicate that ZAIN customers are satisfied with the services. 


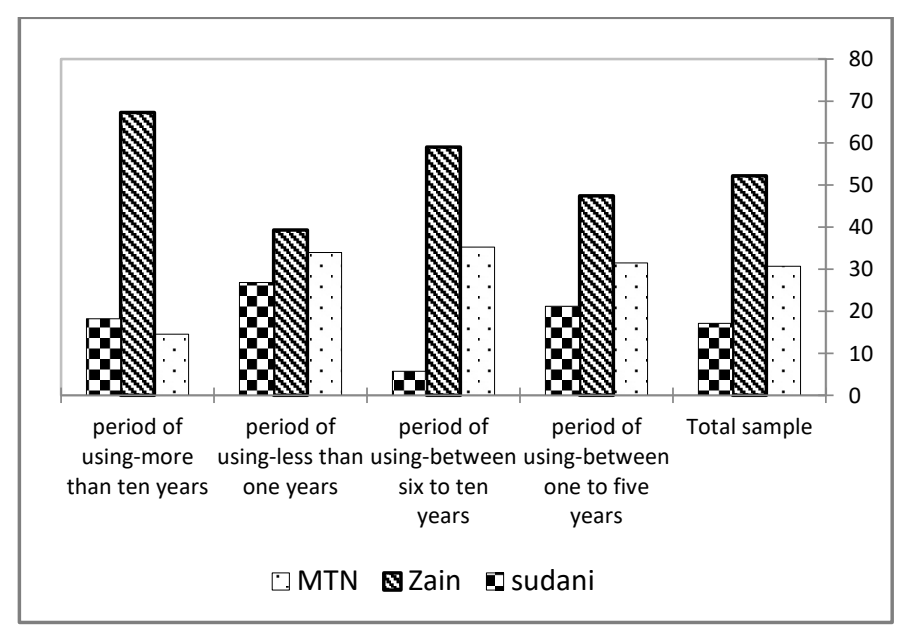

Figure 4: Type of SIM card used with different periods

Figure 5 shows the speed of the internet for all telecommunication company. The obtained results show that ZAIN Company is the best in internet service compared to the other two companies.

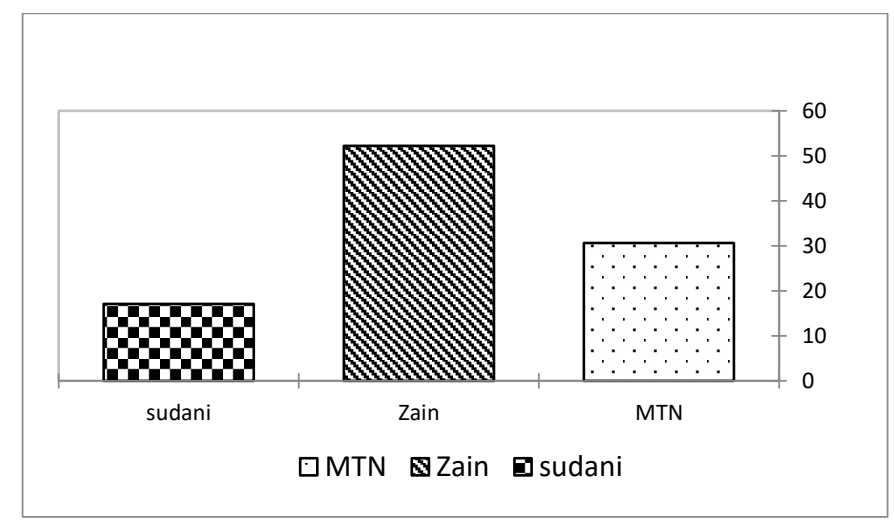

Figure 5: Companies provide good internet speed

Figure 6 show the offers and affordable prices for the three companies, ZAIN is identified as the best company in offers as well as in prices. 


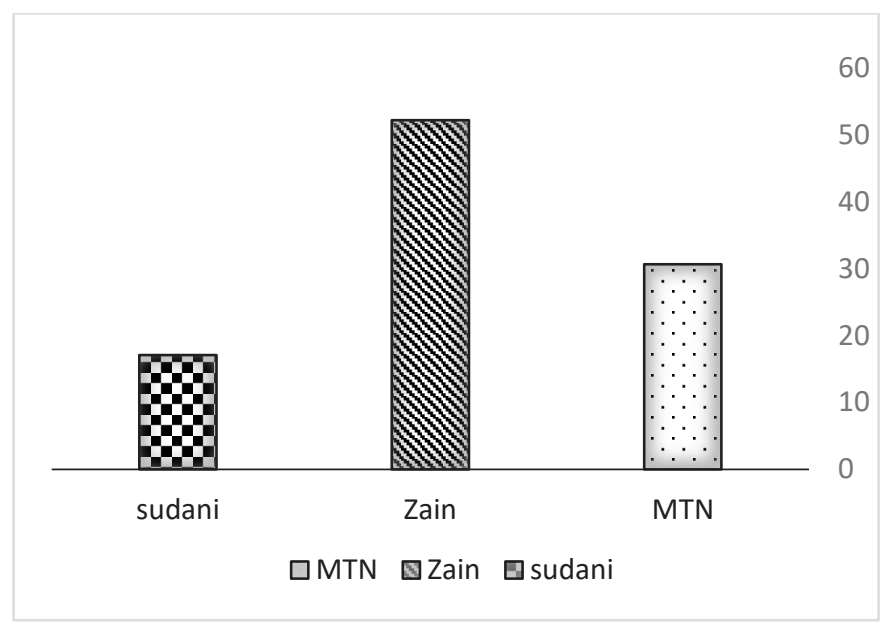

Figure 6: Companies provide best service and affordable price

Figure 7 show the stability of the network connection for calls. ZAIN also represent the best company in providing those services.

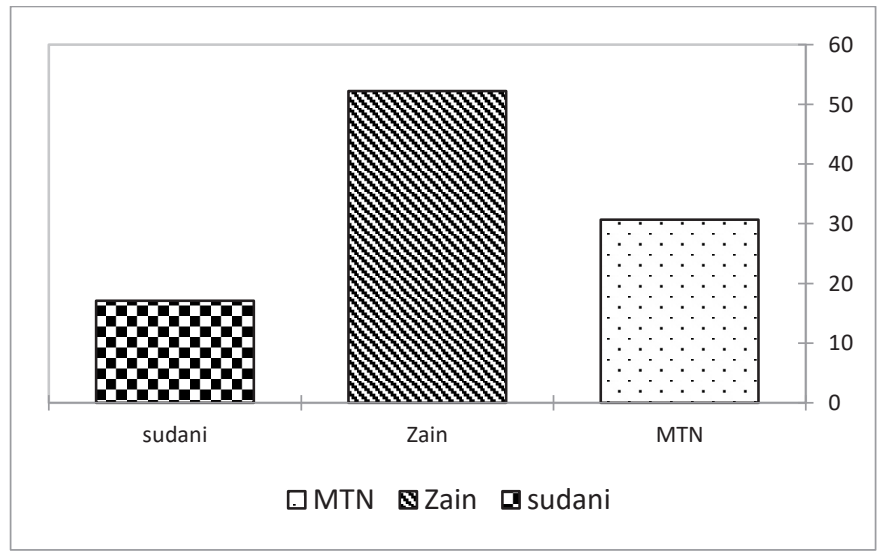

Figure 7: Company provide good quality call

It is clearly seen from the obtained result that Zain as a first company established the mobile service in Sudan have a highest rate in all services compared to the other two companies MTN and Sudani. Moreover, these companies selling similar products in the same marketspace and new entrants into market, influenced by the number and size of active competitors, each competitor's market share, competitors' profitability, customers' intimacy, and rivalry among existing competitors, such as pricing has huge impact on these findings. Many algorithms have been used for discovering the frequency and association rule [11] in the past. Most of the studies prove that the two reputed algorithms used for frequent pattern and association rules are Apriori and FP-Growoth. But in our case Apriori, it performs better because it works for spare and medium datasets and FP-Grwoth need to build tree then discover the freuquent item sets. Hence it is concluded that based on the results obtained, Apriori algorithm perfroms better. In addtion our objectives in this study is limited to discovering the frequent pattern as well as analyzing the associations. 


\section{Conclusion}

The study aimed at measuring the satisfaction of customers with the telecom companies in Sudan. A survey questionnaire was designed, and the data collected from customers have been analysed to find the frequent patterns and association rule mining techniques. The obtained results show that Zain Sudan performs comparatively better than the MTN and Sudani in different kind of services provided to the customers in terms of quality of call, internet speed, and duration of using the SIM card.

As a future work, the study can be extended to cover other states beside Khartoum that may be affected by the coverage and the quality of services. Also, other kind of data mining techniques can be applied to find out which algorithm provides more accurate results.

\section{References}

[1] O. S. G. P. IRENE BUKARI, IRENE HAGAR, "an Assessment of Customer Satisfaction in the Telecom Sector of Ghana: a Case Study of Vodafone Ghana Limited,'Ir.Csuc.Edu.Gh:8080/Xmlui/Handle/123456789/247, vol. 1, no. June, p. 73, 2013.

[2] L. Wong and L. Zhang, Selected Topics in Post-Genome Knowledge, no. January 1996. 2004.

[3] K. Arutchelvan and R. Periyasamy, "Cancer Prediction System Using Datamining Techniques," pp. 1179-1183, 2015.

[4] A. H. Al-hashedi and S. A. Abkar, "The Impact of Service Quality Dimensions on Customer Satisfaction in Telecom Mobile Companies in Yemen," vol. 7, no. 4, pp. 186$193,2017$.

[5] E. Laukkanen, J. Itkonen, and C. Lassenius, "This is an electronic reprint of the original article. This reprint may differ from the original in pagination and typographic detail . Problems, Causes and Solutions When Adopting Continuous Delivery - A Systematic Literature Review Problems , causes a," vol. 2017, pp. 3-4, 2017.

[6] C. Marco and C. Rendón, "Proposed Model for Measuring Customer Satisfaction with Telecommunications Services Andrés Vásquez Martha Benjumea-Arias Alejandro Valencia-Arias," vol. 8, no. 2, pp. 15-25, 2017.

[7] B. Natarajan, P. Daniels, S. Shine, and S. Rizwan, “An Analysis of Customer Satisfaction with Service Delivery of Botswana Telecommunications Corporation ( BTC ) Mobile Network: A Case Study in Francistown," Int. J. Multidiscip. Curr. Res., vol. 4, 2016.

[8] P. Report, "Factors Influencing Customer Satisfaction In Mobile Telecommunications Industry of Nepal Business Research Project Report By," 2014.

[9] W. M. Olatokun and F. O. Ojo, "Influence of service quality on consumers' satisfaction with mobile telecommunication services in Nigeria," Inf. Dev., vol. 32, no. 3, pp. 398408, 2014.

[10] L. Li et al., "Data mining techniques for cancer detection using serum proteomic profiling," Artif. Intell. Med., vol. 32, no. 2, pp. 71-83, 2004.

[11] S. Solanki and N. Soni, "A Survey on Frequent Pattern Mining Methods Abstract," vol. $\mathrm{X}$, no. X. 